Background: Still's disease is a systemic auto-inflammatory disease with a pediatric form, SJIA, and an analogous condition in adults, adult-onset Still's disease (AOSD). The role of interleukin-1 (IL-1) in the pathophysiology of Still's disease is well established. Canakinumab, a monoclonal antibody against IL-1B, is approved to treat patients with Still's disease in Europe (SJIA and AOSD) and the United States (SJIA).

Objectives: To study the efficacy of canakinumab in SJIA patients categorized by age, we performed an intention-to-treat analysis of pooled data from 5 clinical trials, as an addition to a previously communicated analysis including 3 of the studies ${ }^{1}$ Methods: The age categories were children ( $2-<12$ years), young adolescents $(12-<16$ years) and older adolescents and young adults $(16-<20$ years). We pooled efficacy results from patients with active disease at baseline treated during a 12-week period with canakinumab ( $4 \mathrm{mg} / \mathrm{kg}$ every 4 weeks), including the presence of intermittent fever, serum concentrations of $C$ reactive protein (CRP), improvement of SJIA (adapted pediatric ACR 30, 70 and 100 responses) and JIA ACR inactive disease status. Safety was assessed by analysis of reported adverse events (AEs).

Results: 302 children, 82 young adolescents and 34 older adolescents and young adults were included in the analysis, with a mean disease duration of 922 , 1708 and 2615 days, respectively. Prior therapy with other biologics was common, with anakinra used in $33 \%, 35 \%$ and $47 \%$ of patients in each group. Disease severity was comparable among groups, with the mean number of active joints ranging from 11.8 to 13.7. Adapted pediatric ACR responses revealed a rapid response to canakinumab, with all groups showing similar rates of responders at most time points (Table 1). In each age group, the proportion of patients with inactive disease progressively increased to Day 57. At all time points after Day 15 , the $16-<20$ years group presented the highest proportion of patients with inactive disease. Median CRP levels decreased from baseline to reach values in the normal range $(<10 \mathrm{mg} / \mathrm{L})$ from Day 29 onwards in the three groups, with improvements more marked in the $16-<20$ years group. The safety profile was similar in the three age groups analyzed, with a lower proportion of $16-<20$ years old patients experiencing serious AEs (28\%) as compared to children (35\%) and young adolescents (42\%).

Table 1. Percentages of patients with Adapted pediatric ACR responses and inactive disease status over time*

\begin{tabular}{ccccc}
\hline & $\begin{array}{c}\text { Time of treatment } \\
\text { (Days) }\end{array}$ & $2-<12$ years $^{\mathrm{a}}$ & $12-<16$ years $^{\mathrm{b}}$ & $16-<20$ years $^{\mathrm{c}}$ \\
\hline ACR30 & 15 & 72.7 & 81.7 & 83.9 \\
$(\%)$ & 29 & 77.5 & 84.1 & 82.4 \\
& 57 & 76.2 & 82.9 & 88.2 \\
ACR70 & 85 & 65.5 & 74.5 & 83.3 \\
$(\%)$ & 15 & 51.5 & 58.3 & 64.5 \\
& 29 & 61.9 & 62.2 & 70.6 \\
ACR100 & 57 & 65.2 & 58.5 & 79.4 \\
$(\%)$ & 85 & 58.6 & 61.8 & 75.0 \\
& 15 & 21.6 & 25.0 & 12.9 \\
Inactive disease & 29 & 29.5 & 30.5 & 35.3 \\
$(\%)$ & 57 & 36.1 & 34.1 & 38.2 \\
& 85 & 34.1 & 30.9 & 33.3 \\
& 15 & 19.0 & 30.0 & 19.4 \\
& 29 & 34.1 & 34.1 & 47.1 \\
& 57 & 39.4 & 36.6 & 55.9 \\
& 85 & 36.7 & 43.4 & 52.2 \\
\hline
\end{tabular}

*Some studies did not include visits at Day 15 and/or 85. For Day 15, 29, 57 and 85 the respective denominators for each age group were: ${ }^{\mathrm{a}} \mathrm{N}=231,302,302,232 ;{ }^{\mathrm{b}} \mathrm{N}=60,82,82$, $55 ;{ }^{\mathrm{c}} \mathrm{N}=31,34,34,24$.

Conclusion: The efficacy and safety profile of canakinumab was consistent in children, adolescents and young adults with SJIA. Since SJIA and AOSD represent pediatric- and adult- onset variants of the Still's disease continuum, these results further support the therapeutic effect of canakinumab $4 \mathrm{mg} / \mathrm{kg}$ every 4 weeks in both children and adults with Still's disease.

References:

[1] Feist et al. Clin Exp Rheumatol. 2018;36(4):668-75

Disclosure of Interests: Pierre Quartier Consultant of: AbbVie, Chugai-Roche, Lilly, Novartis, Sanofi, Sobi, Speakers bureau: AbbVie, BMS, Chugai-Roche, Novartis, Pfizer, Sobi, Eugen Feist Consultant of: Novartis, Roche, Sobi, Lilly, Pfizer, Abbvie, BMS, MSD, Sanofi, Speakers bureau: Novartis, Roche, Sobi, Lilly, Pfizer, Abbvie, BMS, MSD, Sanofi, Daniel J Lovell Consultant of: Abbott (consulting and PI), AbbVie (PI), Amgen (consultant and DSMC Chairperson), AstraZeneca, Boehringer Ingelheim, Bristol-Myers Squibb (PI), Celgene, Forest Research (DSMB Chairman), GlaxoSmithKline, Hoffman-La Roche, Janssen (co-PI), Novartis (consultant and PI), Pfizer (consultant and $\mathrm{PI})$, Roche (PI), Takeda, UBC (consultant and PI), Wyeth, Employee of: Cincinnati Children's Hospital Medical Center, Speakers bureau: Wyeth, Hiroaki Umebayashi: None declared, Nicolino Ruperto Grant/research support from: Bristol-Myers Squibb, Eli Lily, F Hoffmann-La Roche, GlaxoSmithKline, Janssen, Novartis, Pfizer,
Sobi (paid to institution), Consultant of: Ablynx, AbbVie, AstraZeneca-Medimmune, Biogen, Boehringer Ingelheim, Bristol-Myers Squibb, Eli Lily, EMD Serono, GlaxoSmithKline, Hoffmann-La Roche, Janssen, Merck, Novartis, Pfizer R-Pharma, Sanofi, Servier, Sinergie, Sobi, Takeda, Speakers bureau: Ablynx, AbbVie, AstraZeneca-Medimmune, Biogen, Boehringer Ingelheim, Bristol-Myers Squibb, Eli Lily, EMD Serono, GlaxoSmithKline, Hoffmann-La Roche, Janssen, Merck, Novartis, Pfizer, R-Pharma, Sanofi, Servier, Sinergie, Sobi, Takeda, Hermine Brunner Consultant of: Hoffman-La Roche, Novartis, Pfizer, Sanofi Aventis, Merck Serono, AbbVie, Amgen, Alter, AstraZeneca, Baxalta Biosimilars, Biogen Idec, Boehringer, Bristol-Myers Squibb, Celgene, EMD Serono, Janssen, Medlmmune, Novartis, Pfizer, and UCB Biosciences, Speakers bureau: GSK, Roche, and Novartis, Cornelia Dunger-Baldauf Employee of: Novartis, Stephanie Noviello Employee of: Novartis, sarah whelan Employee of: Novartis DOI: 10.1136/annrheumdis-2020-eular.3759

\section{OP0293 USE OF WHOLE-BODY MAGNETIC RESONANCE TO IDENTIFY POTENTIAL DIAGNOSTIC CLUES IN CHILDREN WITH FEVER OF UNKNOWN ORIGIN (FUO)}

S. Signa ${ }^{1,2}$, R. Caorsi ${ }^{1}$, G. Stagnaro ${ }^{3}$, F. Minoia $^{4}$, P. Picco ${ }^{4}$, A. Ravelli ${ }^{2,4}$, M. B. Damasio ${ }^{3}$, M. Gattorno ${ }^{1} .{ }^{1}$ IRCCS Istituto Giannina Gaslini, UOSD Centro Malattie Autoinfiammatorie ed Immunodeficienze, Genoa, Italy; ${ }^{2}$ University of Genoa, DINOGMI, Genoa, Italy; ${ }^{3}$ IRCCS Istituto Giannina Gaslini, UOC Radiologia, Genoa, Italy; ${ }^{4}$ IRCCS Istituto Giannina Gaslini, UOC Clinica Pediatrica e Reumatologia, Genoa, Italy

Background: Whole-body magnetic resonance imaging (WBMRI) is a fast and accurate method to detect diseases throughout the entire body without exposure to ionizing radiation. Possible emerging applications for this technique include rheumatologic field and evaluation of fever of unknown origin (FUO).

Objectives: To evaluate the ability of WBMRI to identify significant potential diagnostic clue (PDC) in patients presenting a non specific inflammatory clinical picture.

Methods: We retrospectively collected cases of pediatric patients followed in a single pediatric rheumatology center who underwent WBMRI between January 2010 and December 2015 for the following indications: i) FUO (temperature greater than $38.3^{\circ} \mathrm{C}$ for more than three weeks or failure to reach diagnosis after one week of investigations), iii) recurrent fever (febrile episodes separated by periods of normal temperature), iii) Inflammation of unknown origin, IUO (an illness of at least 3 weeks' duration, with raised inflammatory markers and fever below $38.3^{\circ} \mathrm{C}$ ).WBMRI studies were acquired with coronal and sagittal planes (slice thickness $5 \mathrm{~mm}$ ) with acquisition of several image sets with automatic direct image realignment after acquisition creating a whole-body scan.Sequences include short $\mathrm{\tau}$ inversion recovery (STIR) and T1-weighted. All studies have been evaluated twice, the second time according to a predefined checklist, defined by an experienced radiologist, considering systematically single /multifocal bone lesion, bone marrow, joint effusion, soft tissues, adenopathies, parenchymal and vessels looking for PDC. We considered as a Potential Diagnostic Clue each alteration of the examined district that can potentially guide the diagnosis. Each alteration found is a PDC. We retrospectively evaluated patients' clinical history and final diagnosis and we classified the PDCs identified during both first evaluation and re-evaluation as: Not useful (the identified PDC did not guide the diagnosis and is not coherent with the final diagnosis), consistent (the identified PDC is congruent with the patient's final diagnosis) or diagnostic (the identification of the considered PDC strongly orient the final diagnosis).

Results: We collected 104 patients who underwent WBMRI; 24 (23\%) of them presenting FUO, 28 (27\%) presenting recurrent fever and $52(50 \%)$ presenting IUO. The mean age of onset symptoms was 6 years and nine months (range: 2 weeks old- 17 years and 6 months). The mean age of execution of WBMRI was 9 years (range: 5 months old- 19 years). After the whole diagnostic work-out a final diagnosis was achieved in 44 patients (42\%).PDCs were identified at the first evaluation in $78 / 104$ cases $(75 \%)$. In 22 cases $(21 \%)$ the identified PDCs were consistent with the diagnosis, whereas in 9 cases $(8.5 \%)$ the identified PDCs were considered diagnostic. Globally we can consider that at first evaluation PDCs were somehow contributory to the diagnosis in 31 cases $(30 \%$; 6 JIA, 7 systemic infections, 5 monogenic inflammatory diseases, 4 ALPS, 2 Goldbloom's Syndrome,2 Vasculitis, 1 eosinophilic fasciitis, 1 hystiocytosis, 3 oncologic diagnosis).Blind re-evaluation of WBMRI allowed the identification of additional PDCs in 52 patients (12 of them previously negative). In 10 cases the PDC found after re- evaluation were consistent with the final diagnosis (2 JIA one infectious disease, one neuroblastoma, 3 ALPS, 1 monogenic inflammatory disease, 1Takayasu arteritis, 1 Goldbloom's syndrome).

Conclusion: WBMRI can be a powerful diagnostic tool in patients with FUO. A predefined checklist increases sensitivity of WBMRI in the identification of PDC. Disclosure of Interests: Sara Signa: None declared, Roberta Caorsi: None declared, Giorgio Stagnaro: None declared, Francesca Minoia: None declared, Paolo Picco: None declared, Angelo Ravelli: None declared, Maria Beatrice 
Damasio: None declared, Marco Gattorno Consultant of: Sobi, Novartis, Speakers bureau: Sobi, Novartis

DOI: 10.1136/annrheumdis-2020-eular.2640

\section{Osteoporosis}

\section{OP0294 DIFFERENTIAL INFLUENCE OF CO-MORBIDITIES ON SITE OF FRAGILITY FRACTURES}

M. Dey ${ }^{1,2}$, M. Bukhari ${ }^{3} .{ }^{1}$ Institute of Ageing and Chronic Disease, University of Liverpool, Musculoskeletal Biology I, Liverpool, United Kingdom; ${ }^{2}$ Aintree University Hospital, Liverpool University Hospitals NHS Foundation Trust, Academic Rheumatology, Liverpool, United Kingdom; ${ }^{3}$ Royal Lancaster Infirmary, University Hospitals of Morecambe Bay NHS Foundation Trust, Rheumatology, Lancaster, United Kingdom

Background: Fragility fractures (FF) can occur at various sites of the skeleton and are associated with multiple risk factors [1]. The prevalence of FF markedly increases with age. As the longevity of the population increases, so will the incidence of FF, and that of associated co-morbidities and risk factors. There are few data on co-morbidities associated with fractures at each site.

Objectives: Identify associations of co-morbidities with sites of FF, by applying cluster analysis.

Methods: We reviewed 28868 patients presenting for BMD estimation at a district general hospital in North West England, 2004-2016. We identified patients who had sustained one or more FF at time of presentation. Site(s) of FF were recorded for each patient, including femur, forearm, humerus, pelvis, ribs, spine, tibia or fibula. The following co-morbidities or treatments were recorded: excess alcohol consumption (previous or current); bisphosphonates; coeliac disease; family history of FF; hormone replacement therapy; hyperparathyroidism; hyperthyroidism; inflammatory bowel disease; polymyalgia rheumatica; rheumatoid arthritis; smoking (previous or current); corticosteroids (previous or current). Cluster analysis was performed on fracture sites and co-morbidities, using Jaccard similarity coefficient, and plotted on a dendrogram. Results were divided into an optimal number of clusters, derived using the elbow and silhouette methods.

Results: 11003 of 28868 patients had sustained one or more FF at time of BMD estimation. Overall, $84.6 \%$ patients were female, mean age $67.5 y e a r s$, and median T-score $-1.12 \mathrm{SD}$. Cluster analysis was performed for FF sites and co-morbidities, with Jaccard similarity coefficients calculated. 4 clusters were identified (Figure 1): FF of forearm $(n=5054)$, tibia/fibula $(n=2617)$, spine $(n=2352)$, associated with family history of FF, smoking, corticosteroids, and bisphosphonate treatment; FF of pelvis $(n=300)$ associated with hyperparathyroidism, PMR, coeliac disease, and HRT; FF of femur $(n=1181)$ and humerus $(n=1131)$ associated with IBD and RA; FF of ribs $(n=1022)$ associated with alcohol and hyperthyroidism.

Conclusion: Cluster analysis demonstrated 4 distinct subgroups of FF sites and associated co-morbidities. To our knowledge, this is the first study applying cluster analysis to evaluate co-morbidities associated with FF sites. Risk factors may influence trabecular more than cortical bone, accounting for the difference in clusters. Knowledge of risk factors associated with FF site subgroups will aid prophylaxis and management in at-risk patients.

References:

[1] Kanis JA. Diagnosis of osteoporosis and assessment of fracture risk. Lancet (London, England). 2002 Jun 1;359(9321):1929-36

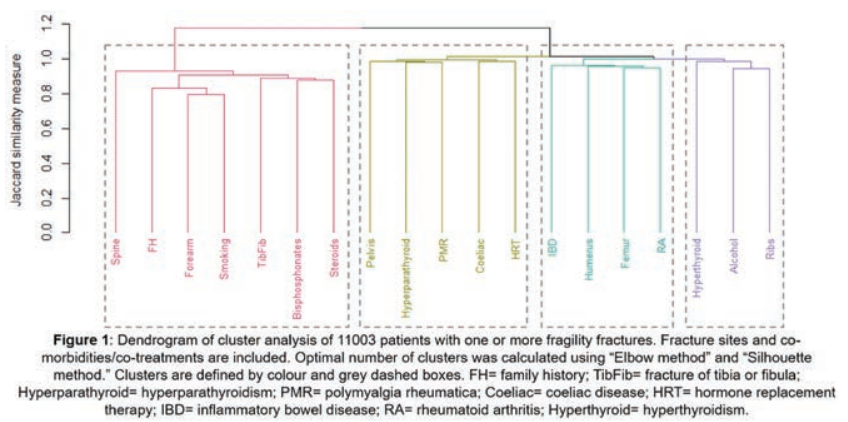

Disclosure of Interests: Mrinalini Dey: None declared, Marwan Bukhari Speakers bureau: Bristol-Myers Squib, UCB celltech, Roche/Chugai, Pfizer, Abbvie, Merck, Mennarini, Sanofi-aventis, Eli-Lilly, Janssen, Amgen and Novartis. DOI: 10.1136/annrheumdis-2020-eular.855

\begin{tabular}{|l|l|}
\hline OP0295 & CORRELATION BETWEEN CORTICAL THICKNESS \\
RELATIVE TO TRANSVERSE DIAMETER ON MID- \\
PORTION OF THIRD METACARPAL BONE AND BONE \\
MINERAL DENSITY IN LUMBAR SPINE AND FEMORAL \\
NECK FOR PATIENTS WITH RHEUMATOID ARTHRITIS
\end{tabular}

I. Yoshii'. ' Yoshii Hospital, Department of Rheumatology and Musculoskeletal Medicine, Shimanto City, Japan

Background: Rheumatoid arthritis (RA) is a determinant risk factor of osteoporosis. BMD is clearly defined as diagnosis criteria of osteoporosis in Japan; that is less than -2.5 with T-score measured with dual-energy X-ray absorptiometry (DXA). The marker of T-score $<-2.5$ is widely used worldwide; however, the testing system is very expensive, preventing its extensive adoption.

Objectives: We tried to evaluate BMD not measuring with DXA, but the othe method that substituting DXA with another X-ray picture of hand that is routinely taken for Sharp/van der Heijde score (SHS) calculation.

Methods: Patient with RA, who met the American College of Rheumatology/ European League Against Rheumatism classification criteria, visited our institute has been routinely calculated SHS in taking X-ray pictures of bilateral hands and feet at first consultation. Cortical thickness was calculated from mid-portion of third metacarpal bone in X-ray picture that was taken for the calculation of SHS as taking cancellous bone diameter of the third metacarpal bone from transverse diameter at the same point. We set Cortical Thickness Ratio (CTR) as cortical thickness relative to transverse thickness (Figure 1). BMD measurements at the lumbar spine (LS) and femoral neck (FN) were obtained, and BMD values are presented as $\mathrm{g} / \mathrm{cm}^{2}$ and T-score showing dissociation of the BMD compared with the mean BMD in healthy 30 -year-old of the same sex with standard deviation was also presented. Patient with RA who underwent SHS calculation and BMD measurement at first consultation was picked up for the study. Relationship between BMD and the other parameters such as sex (male/ female), age, disease duration (years), ACPA titer, RF titer, body mass index (BMI), CTR, the HAQ score, DAS28-CRP, SHS, PS-VAS, tartrate-resistant acid phosphatase-5b value (TRACP-5b), previous treatment for osteoporosis and RA before initial consultation ( $\mathrm{pTx}$ OP and $\mathrm{pTx}$ RA) (Yes/No) at initial consultation was evaluated with linear regression analysis.

T-score $<-2.5$ was statistically evaluated with binary regression analysis for the parameters that demonstrated significant correlation in multivariate linear regression analysis

Then, Cut-off index (COI) of CTR for the BMD represented with T-score $<-2.5$ for both of LS and FN was evaluated with Receivers Operation Characteristics technique (ROC). Sensitivity, specificity, area under curve, odds ratio with $95 \%$ confidence interval $(95 \% \mathrm{Cl})$ for T-score $<-2.5$ was also calculated.

Results: A total of 300 patients were picked up for the study. BMDs were 0.867 and 0.682 with 0.203 and 0.143 for standard deviations, that means T-score was -1.93 and -1.86 with 1.64 and 1.15 for standard deviations in LS and FN, respectively. Mean transverse width of third metacarpal bone was $7.3 \mathrm{~mm}$ and thickness of the cortex was $2.00 \mathrm{~mm}$, so CTR was 0.279 in average and 0.124 for standard deviation.

In linear regression analysis, BMD in LS demonstrated significant correlation with sex, CTR, and DAS28-CRP, while BMD in FN demonstrated significant correlation with sex, age, and CTR.

In binary regression analysis, CTR and DAS28-CRP demonstrated significant positive correlation with T-score $<-2.5$ in LS, while age and CTR demonstrated significant correlation in $\mathrm{FN}$.

In ROC, cut-off index of CTR was 0.25 in both of LS and FN, and sensitivities demonstrated $67.9 \%$ and $76.1 \%$, and specificity demonstrated $83.0 \%$ and $81.6 \%$ in LS and FN, respectively. Area under curve was 0.78 and 0.81 with 4.17 (95\%Cl: $2.51-6.92)$ and $4.90(95 \% \mathrm{Cl}: 2.75-8.73)$ of odds ratios for $\mathrm{LS}$ and $\mathrm{FN}$, respectively (Figure 2).

Conclusion: Results of this cross-sectional study encourages our hypothesis that thickness of cortical bone relative to full thickness in the long bone reflects BMD. CTR correlated with BMD in both of LS and FN. CTR of third metacarpal bone was suggested that has close correlation with BMD in both LS and FN CTR could be strong candidate marker for screening of osteoporosis in patient with RA with the index less than 0.25 